\title{
Inferior Olive Oscillations Gate Transmission of Motor Cortical Activity to the Cerebellum
}

\author{
Sarah P. Marshall and Eric J. Lang \\ Department of Physiology and Neuroscience, New York University, School of Medicine, New York, New York 10016
}

Inferior olivary (I0) neurons display spontaneous oscillatory activity, yet the importance of these oscillations for shaping the responses of this system to its afferents is uncertain. We used multiple electrode recording of crus 2a Purkinje cell complex spikes (CSs) in ketamine-xylazine-anesthetized rats to investigate olivocerebellar responses to activation of motor cortico-olivary pathways. Trains of electrical stimuli were applied to the motor cortex at frequencies between 4 and $30 \mathrm{~Hz}$. Various frequency-response curves were observed, with the most common types being unimodal with a maximum at $9.5 \pm 2.3 \mathrm{~Hz}$ and bimodal with peaks at $8.9 \pm 1.0$ and $15.1 \pm 1.3 \mathrm{~Hz}$. To determine whether IO oscillatory properties underlie the resonance peaks in the frequency-response curves, apamin and charybdotoxin were injected into the IO. These toxins, which weaken and enhance spontaneous IO oscillations, respectively, had corresponding effects on the sharpness of resonance peaks. Next, the variation of CS entrainment patterns with frequency was investigated to characterize the nature of the IO oscillator. Low-frequency $(4 \mathrm{~Hz})$ stimulation was relatively ineffective in entraining CS activity. Between 4 and $30 \mathrm{~Hz}$, two predominant entrainment patterns emerged. For low-frequency $(4-6 \mathrm{~Hz})$ and high-frequency $(17-30 \mathrm{~Hz})$ ranges, a 1:2 entrainment dominated, whereas in the intermediate range $(6-17 \mathrm{~Hz}), 1: 1$ entrainment was most prevalent. These results indicate that I0 neurons respond as nonlinear oscillators to afferent signals.

Key words: oscillator; complex spike; Purkinje cell; cerebellum; motor cortex; gating

\section{Introduction}

The olivocerebellar system generates rhythmic and synchronous complex spike (CS) activity in Purkinje cells (Sasaki et al., 1989; Lang et al., 1999). This phenomenon is thought to result from electrotonic coupling of inferior olivary (IO) neurons and the ability of these cells to generate oscillatory activity. That IO neurons are spontaneous oscillators is supported by several lines of evidence: IO neurons recorded from brain slices (in which their afferent input has been effectively eliminated) display subthreshold oscillations in their membrane potential (Llinás and Yarom, 1981a,b, 1986; Benardo and Foster, 1986; Lampl and Yarom, 1997); rhythmic CS activity can be altered by intra-IO injections of apamin and charybdotoxin, antagonists of the smallconductance (SK) and large-conductance (BK) types of $\mathrm{Ca}^{2+}$ dependent $\mathrm{K}^{+}$channels, respectively (Lang et al., 1997); and rhythmic CS activity not only remains but actually becomes more prevalent after block of glutamatergic and GABAergic input to the IO in the intact animal (Lang et al., 1996; Lang, 2001).

Relatively few studies, however, have addressed the issue of how these oscillatory properties shape the olivocerebellar system's interactions with activity from other brain areas, and most of these have investigated its free-running response after a single

Received Jan. 20, 2004; revised Nov. 8, 2004; accepted Nov. 8, 2004.

This work was funded by grants from the National Institutes of Health-National Institute of Neurological Disorders and Stroke (NS37028) and the National Science Foundation (IBN-9808353). We thank R. Bing for preparation of histological specimens, K. Maxwell for help with the recordings, and D. Paré for comments on this manuscript.

Correspondence should be addressed to Dr. E. J. Lang, Department of Physiology and Neuroscience, New York University Medical Center, 550 First Avenue, New York, NY 10016. E-mail: lange01@med.nyu.edu.

D01:10.1523/JNEUROSCI.3907-04.2004

Copyright $\odot 2004$ Society for Neuroscience $\quad$ 0270-6474/04/2411356-12\$15.00/0 perturbation. For example, the ongoing subthreshold oscillation of IO neurons can cause their response to synaptic activity to be phase advanced or delayed, depending on the phase of the cycle at which the activity arrives (Lampl and Yarom, 1993). Alternatively, a strong stimulus will reset the IO oscillation cycle and produce a damped oscillatory CS or IO spike response (Crill, 1970; Bloedel and Ebner, 1984; Llinás and Sasaki, 1989). These studies suggest that the oscillatory properties of IO neurons are an important determinant of how the olivocerebellar system interacts with other brain regions, yet they do not address the question of how IO neurons might interact with an ongoing oscillatory signal, such as might arise from the motor cortex (MCtx).

Indeed, pathways originating from the MCtx provide significant functional input to the IO, because electrical stimulation of MCtx readily evokes CS activity (Provini et al., 1968; Allen et al., 1974; Lang, 2001). Moreover, MCtx displays behavior-related synchronous oscillatory activity over a wide frequency range that spans at least 4-50 Hz (Steriade and Hobson, 1976; Bouyer et al., 1987; Ribary et al., 1991; Murthy and Fetz, 1992, 1996; Sanes and Donoghue, 1993; Nicolelis et al., 1995). Thus, these two systems form an arrangement in which oscillatory activity from one system (MCtx) could drive the second oscillatory system (olivocerebellar). To investigate the interaction of these two neuronal systems, we characterized the CS response patterns evoked by MCtx stimulation trains of different frequencies. The observed response patterns show that the IO acts as a nonlinear oscillator that preferentially responds to input within a relatively small frequency range surrounding $10 \mathrm{~Hz}$. 


\section{Materials and Methods}

Experiments were performed in accordance with the National Institutes of Health guidelines for the care and use of laboratory animals. Experimental protocols were approved by the Institutional Animal Care and Use Committee of New York University, School of Medicine.

\section{Surgical and electrode implantation procedures}

Adult female Sprague Dawley rats (225-300 gm) were anesthetized with an initial intraperitoneal injection of ketamine $(100 \mathrm{mg} / \mathrm{kg})$ and xylazine $\left(8 \mathrm{mg} / \mathrm{kg}\right.$ ). Supplemental anesthesia (ketamine, $\sim 260 \mu \mathrm{g} \cdot \mathrm{kg}^{-1} \cdot \mathrm{min}^{-1}$; xylazine, $\sim 4 \mu \mathrm{g} \cdot \mathrm{kg}^{-1} \cdot \mathrm{min}^{-1}$ ) was started $1-2 \mathrm{hr}$ after the initial injection as a continuous intravenous injection via a femoral vein catheter to maintain a constant anesthetic depth. A tracheal tube was inserted to allow ventilation of the animal along with delivery of supplemental oxygen. Temperature was monitored rectally and maintained at $36-37^{\circ} \mathrm{C}$ by use of an electric heating pad.

The experiments required obtaining multi-electrode recordings from crus 2a Purkinje cells during stimulation of the contralateral MCtx. To access these areas, the animal was placed in a stereotaxic apparatus, and the skin, muscle, and bone overlying the cerebellum and MCtx were resected. The MCtx was covered with a piece of Gelfoam that was soaked with Ringer's solution. A platform was positioned on the surface of crus $2 \mathrm{a}$ and cemented into place. The platform, which served to stabilize the microelectrodes and protect the surface of the brain, was composed of an electron microscope grid encased in silicone rubber and fixed to tungsten metal rods. Glass microelectrodes filled with a 1:1 solution of $\mathrm{NaCl}$ and glycerol ( $\sim 2 \mu \mathrm{m}$ tip diameter) were inserted individually through the grid using a joystick-controlled three-axis micromanipulator (Burleigh Instruments, Victor, NY). A single electrode was attached to the micromanipulator with a wax droplet and pushed through the rubber platform into the cerebellar cortex. CS activity was isolated in the molecular layer, at a depth of 75-100 $\mu \mathrm{m}$ below the cortical surface, where simple spike activity is absent. Once CS activity had been isolated, the wax was melted to release the electrode, and the manipulator was attached to another electrode. Electrodes were spaced $\sim 250 \mu \mathrm{m}$ apart such that they formed a rectangular array of up to 4 mediolaterally running rows and 10 rostrocaudally directed columns.

\section{MCtx stimulation protocol}

MCtx stimulation was delivered using a bipolar electrode inserted to a depth of $\sim 1 \mathrm{~mm}$ into the vibrissal region of the MCtx contralateral to the cerebellar recording area. An isolated pulse generator (A-M Systems, Carlsborg, WA) was used to deliver 4-sec-long stimulus trains of 100 $\mu$ sec current pulses at various frequencies ranging from 4 to 25 or $30 \mathrm{~Hz}$. Stimulus trains were delivered at $30 \mathrm{sec}$ intervals throughout a $20-40$ min recording period. During a recording period, stimulus intensity (0.1-1 mA) was held constant, whereas the frequency was varied between individual stimulus trains such that a total of 5-10 trains were delivered at each test frequency. Stimuli typically evoked CS activity in crus 2a and whisker movements either bilaterally or contralateral to the stimulation site. Thus, to prevent stimulus-evoked movements and the resulting sensory feedback, the paralytic gallamine triethiodide (Flaxedil) was administered intraperitoneally, and the animal was placed on a respirator just before the recording session.

\section{Injection protocol}

In some experiments, apamin (10 $\mu \mathrm{M}$; Sigma-Aldrich, St. Louis, MO), iberiotoxin ( $2 \mu \mathrm{M}$; Sigma-Aldrich), or charybdotoxin (ChTx) $(2 \mu \mathrm{M}$; Sigma-Aldrich) was injected into the IO to modify the oscillatory properties of IO neurons that project to crus $2 \mathrm{a}$. To position the injection pipette correctly, a recording electrode was lowered from the dorsal surface through the brainstem until IO activity that was correlated with CS activity recorded from crus 2 a was found. The stereotaxic coordinates of this location were noted, the microelectrode was withdrawn, and an injection pipette was inserted to the same coordinates.

All drugs were dissolved in Ringer's solution containing (in $\mathrm{mM}$ ): 126 $\mathrm{NaCl}, 26 \mathrm{NaHCO}_{3}, 3 \mathrm{KCl}, 1.2 \mathrm{KH}_{2} \mathrm{PO}_{4}, 1.6 \mathrm{MgSO}_{4}, 2 \mathrm{CaCl}_{2}, 5 \mathrm{HEPES}$, and 15 dextrose. Drugs were delivered by continuous pressure injection at a rate of $0.1-0.2 \mu \mathrm{l} / \mathrm{min}$ that was controlled by a microdialysis pump (CMA Microdialysis, North Chelmsford, MA). The pump drove a sy- ringe that was connected to the injection pipette via polyethylene tubing. After an injection was started, spontaneous CS activity was monitored for the characteristic changes associated with the injected drug [apamin, increased firing rate and decreased rhythmicity; ChTx, increased firing rate and increased rhythmicity (Lang et al., 1997)]. Once such changes were observed and the new activity pattern had stabilized, spontaneous CS activity was recorded for $20 \mathrm{~min}$ followed by recording of CS responses evoked by MCtx stimulation.

\section{Data acquisition}

The recording system (MultiChannel Systems, Reutingen, Germany) consisted of 128 main amplifiers and corresponding preamplifier stages (total gain $=1000,0.2-8.0 \mathrm{kHz}$ bandpass filters) and a dual-processor personal computer. The sampling rate was set to $25 \mathrm{kHz}$ per channel. For each channel, a single-level voltage threshold was set to detect spikes. Both the times of the spikes and their waveforms were recorded. The activity from individual amplifier channels was monitored on a digital oscilloscope and a virtual oscilloscope built using the MCRack software (MultiChannel Systems). In addition, a matrix panel for monitoring the spike activity from all channels simultaneously was generated using the MCRack software. In this panel, the spatial location of each electrode was mapped to a specific box in the matrix corresponding to its relative location on the brain surface. When CS activity of a particular electrode crossed the threshold for discrimination, the box on the panel that corresponded to the spatial location of the electrode on crus 2a flashed. Observation of the panel thus allowed continuous monitoring of the level of CS activity in all electrodes. Any unusual activity detected in the matrix panel was investigated directly using the oscilloscopes. In addition, the effects of the drug injections could be readily detected on-line by changes in the patterns of activity in the matrix panel.

\section{Data analysis}

Spike sorting and data analysis were performed with custom template matching and analysis programs written in Igor Pro (Wavemetrics, Lake Oswego, OR).

Measurement of spontaneous CS rhythmicity. To assess the strength of oscillatory CS activity in single cells, autocorrelograms with time bins of 5 msec were constructed. To quantify the strength of CS oscillations, the following parameters of the autocorrelograms were obtained: oscillation frequency, number of significant peaks, height of the primary peak as a percentage of the total number of spikes, and rhythm index (RI) (Sugihara et al., 1995). Whether a peak or valley was significant was determined by the following procedure. First, the SD of the histogram was measured at time lags of 2000-2500 msec, where oscillatory activity was normally absent and random fluctuations dominated. Next, the average bin height from 50 to $1000 \mathrm{msec}$ was calculated to obtain the local baseline of the autocorrelogram. To be considered significant, peaks in the autocorrelogram had to be $>2$ SDs above the local baseline. The highest bin with a latency between 50 and $500 \mathrm{msec}$ significantly above baseline was considered the primary peak, and its reciprocal was defined as the oscillation frequency. For higher-order peaks to be considered significant, they had to be 2 SDs above baseline and occur at a latency from the preceding peak that was equal to latency of the primary peak $\pm 10 \mathrm{msec}$. For valleys to be significant, they had to occur at a latency from the preceding peak equal to half the latency of the primary peak $\pm 10 \mathrm{msec}$ and be $>1 \times \mathrm{SD}$ below the average bin height, or the difference in height between the preceding peak and the valley had to exceed 2 SDs. Failure to find a significant peak terminated the search for significant peaks and valleys.

The RI was determined with the following formula:

$$
\mathrm{RI}=\sum_{\mathrm{i}}\left(a_{\mathrm{i}}+b_{\mathrm{i}}\right) i=1,2, \ldots,
$$

in which the $a_{\mathrm{i}}$ and $b_{\mathrm{i}}$ are equal to the absolute values of the normalized autocorrelation coefficients (Pearson's $r$ ) of the significant peaks $\left(a_{\mathrm{i}}\right)$ and valleys $\left(b_{\mathrm{i}}\right)$ in the autocorrelogram. Thus, the RI reflects both the amplitude and duration of the oscillatory activity. In autocorrelograms that had no statistically significant peaks and valleys, the RI was zero. In these cases, or when the RI was $<0.01$, the autocorrelation was regarded as nonoscillatory, and the oscillation frequency was not determined. The value of 0.01 
was chosen because autocorrelograms with RI values less than this were subjectively judged not to have discernable peaks.

Analysis of frequency dependence of MCtxevoked CS activity. The probability of MCtx stimuli evoking CS responses was measured by constructing peristimulus time histograms (PSTHs). The PSTHs had time bins of $2 \mathrm{msec}$, and the responses were expressed as a percentage of the number of stimuli used to construct the PSTH. The height of the highest bin between 15 and $30 \mathrm{msec}$ after stimulation was used as a measure of the response probability. This method was used despite its bias to underestimate the true probability, i.e., the peak in the PSTH was usually spread over several bins, because alternative measures, such as peak area, introduce other ambiguities such as defining the start and end bins of the response peak and produced similar results (data not shown).

To investigate the resonance characteristics of the CS responses, a frequency-response curve was generated for each cell by plotting the response probability as a function of stimulus frequency. These curves were characterized with respect to peak (resonant) frequency $\left(f_{\mathrm{p}}\right)$, peak amplitude from the $x$-axis, and half-area width. The half-area width was calculated as the difference $f_{2}-f_{1}$, such that $f_{1}<f_{\mathrm{p}}<f_{2}$, and half the area under the frequency-response curve was contained between $f_{1}$ and $f_{2}$. To obtain $f_{1}$ and $f_{2}$, the absolute differences between each frequency and $f_{\mathrm{p}}$ were set equal to each other and increased incrementally until half the curve area fell between $f_{1}$ and $f_{2}$. If one of the limits of the experimentally tested frequency range $(4-25$ or $30 \mathrm{~Hz})$ was reached before half the area under the curve was contained, the value of one frequency was fixed at this limit, and the difference between $f_{\mathrm{p}}$ and the other one was increased further as required to contain half the area. In general, $f_{1}$ and $f_{2}$ did not occur at a measured data point and so each was obtained by linear interpolation between the data points that surrounded it.

In addition to those parameters obtained from the raw data response curves, we used ones obtained by fitting a Gaussian to each unimodally shaped response curve and to each peak of a bimodally shaped response curve. All fits were assessed using a $\chi^{2}$ goodness of fit test with a 0.05 level for rejecting the fit (Wonnacott and Wonnacott, 1977). The degrees of freedom were equal to the number of data points in the curve -4 (the number of parameters used in the fit) -1 . The four parameters obtained directly from the curve fit procedure were as follows: peak frequency $\left(f_{\mathrm{G}}\right)$, asymptote of the Gaussian, peak amplitude with respect to the asymptote of the Gaussian, and half width at 1/e of the peak amplitude. Two additional parameters were derived from these primary ones: the width, simply defined as twice the half width, and the total peak amplitude, defined as the sum of peak amplitude and asymptote. Last, the regression equation was used to calculate the quality factor, a measure of the relative sharpness of the resonance, which is defined as

$$
\frac{f_{\mathrm{G}}}{\left|f_{\mathrm{a}}-f_{\mathrm{b}}\right|}
$$

where $f_{\mathrm{a}}$ and $f_{\mathrm{b}}$ are the frequencies at which the response curve equals $1 / \sqrt{ } 2$ of the peak amplitude.

Population frequency-response curves were generated from the individual cell frequency-response curves either by simply averaging them or by first normalizing the individual cell curves such that the area under every curve was equal to 1 , and then averaging. These population curves were characterized using the same parameters described for the individual cell curves described above. Similar results were obtained using the normalized and non-normalized averages, so only the latter are reported in Results.

\section{Statistical tests}

Unless specified otherwise, mean values are given with their SD. Student's $t$ test, paired or unpaired as appropriate, was used for comparison

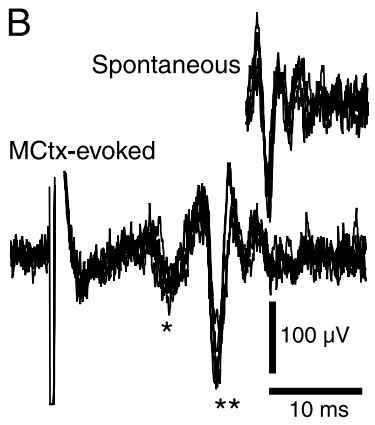

C



of population means. For testing whether a correlation coefficient (Pearson's $r$ ) was statistically different from zero, $r$ was first converted to Fisher's $z^{\prime}\left(z^{\prime}=0.5[\ln (1+r)-\ln (1-r)]\right)$ because $r$ is not normally distributed, whereas $z^{\prime}$ is normally distributed with an $\mathrm{SE}\left(\mathrm{SE}_{z^{\prime}}\right)$ of $1 /(n-$ $3)^{1 / 2}$ (Snedecor and Cochran, 1989). A $z$-score was then computed as $\left(z^{\prime}\right.$ $-0) / \mathrm{SE}_{\mathrm{z}^{\prime}}$ and used to obtain the two-tailed $p$ value.

\section{Histology}

After the recording sessions, the injection pipette was left in place, and its solution was replaced with one containing alcian blue dye. A small quantity $(\sim 0.1 \mu \mathrm{l})$ of dye was then injected to label the pipette tip location. The animal was then perfused intracardially with $0.9 \%$ saline followed by $10 \%$ formalin. The brain was removed and placed in $10 \%$ formalin overnight followed by $30 \%$ sucrose formalin until the brain sank. Coronal slices $(60 \mu \mathrm{m}$ thick) were cut on a freezing microtome and counterstained with cresyl violet to confirm the location of the injection site (see Fig. 1A).

\section{Results}

Simultaneous extracellular recordings of CS activity were obtained from crus 2a Purkinje cells in anesthetized rats using a multiple electrode recording technique. Spontaneous CS activity was recorded from 484 Purkinje cells ( $n=24$ animals; range of 3-34 cells per animal). MCtx-evoked CSs were recorded from a subset of these cells ( $n=420$ cells; 21 animals). Both spontaneous and evoked CSs consisted of high-frequency bursts of two to four spikes (Fig. $1 B$ ). In each experiment, spontaneous CS activity was recorded for an initial 20 min session to determine its basic characteristics for comparison with those of MCtx-evoked CSs. The average spontaneous CS firing rate was $1.0 \pm 0.9 \mathrm{~Hz}$; however, autocorrelograms of most cells displayed peaks at $\sim 100 \mathrm{msec}$ intervals, indicating the presence of an $\sim 10 \mathrm{~Hz}(9.6 \pm 2.9 \mathrm{~Hz})$ rhythmicity (Fig. 1C). To investigate the influence of this rhythmicity on olivocerebellar responses to afferent activity, we analyzed the frequency dependence of MCtx-evoked CS activity patterns.

\section{Preferential transmission of $\sim \mathbf{1 0} \mathrm{Hz}$ afferent activity by the olivocerebellar system}

Stimulus trains, $4 \mathrm{sec}$ in duration, consisting of $100 \mu \mathrm{sec}$ current pulses were delivered to MCtx at frequencies ranging from 4 to 30 $\mathrm{Hz}$. Individual stimuli during such trains evoked CSs at latencies of 15-25 msec, as shown for one Purkinje cell in Figure $2 A$. The highest bin of the PSTH in this latency range was used as a measure of the response probability at each stimulus frequency. These 
A


201
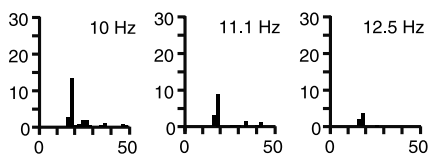

$\therefore$ Time (ms)
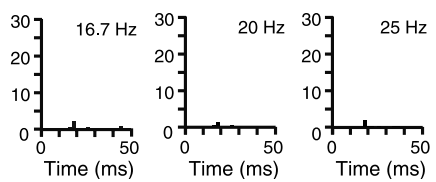

Figure 2. The 10 preferentially transmits $\sim 10 \mathrm{~Hz}$ afferent activity from MCtx. A, PSTHs of CS activity evoked by MCtx stimulus trains of different frequencies in one Purkinje cell. Each PSTH shows the percentage of stimuli that evoked a CS response for stimulus trains of a particular frequency. Stimulus frequency is indicated by the value in the top right corner of each PSTH. PSTH bins are 2 msec. $B$, Unimodal frequency-response curve. The frequency-response curve was generated by plotting the peak responses of the PSTHs shown in $A$ as a function of stimulus frequency. Dashed trace is the Gaussian fit to the data.


Figure 3. Additional varieties of frequency-response curves. $A$, A unimodal frequency-response curve, the most commonly observed type of curve. The dashed line shows the Gaussian fit to the data. $B, C$, Examples of unimodal curves with a highfrequency tail $(B)$ and a low-frequency tail $(C)$. D, Bimodal response curve. Dashed lines show Gaussian fits to the primary and secondary peaks of the curve. $E, F$, Steadily declining $(E)$ and multimodal $(F)$ response curves that were placed into the 0 ther category. $G-I$, Population frequency-response curves from three experiments ( $n=17,24$, and 29 cells) with a unimodal $(G)$, bimodal $(H)$, and multipeaked $(I)$ shape. Error bars represent 1 SD.

response probabilities were then plotted against stimulus frequency for each cell (Fig. $2 \mathrm{~B}$ ). Most of the resulting frequencyresponse curves displayed higher response probabilities for intermediate stimulus frequencies than for those at either extreme of the tested range.

Nevertheless, there was considerable variability in the shape of the curves, as illustrated in Figure $3 A-F$. The large majority of curves could be placed into one of two classes according to their shape: unimodal ( $n=266 ; 63.3 \%$ of total) (Fig. $3 A-C$ ) or bimodal ( $n=110 ; 26.2 \%$ of total) (Fig. $3 D)$. The remaining curves were placed in a heterogeneous "other" group ( $n=44 ; 10.5 \%$ of total). The latter class consisted of curves with various shapes, including those that decreased progressively with frequency (Fig. $3 E$ ) or had three or more peaks with no single dominant one (Fig. 3F).

Curves within the unimodal category could be subdivided further according to their tail pattern. Simple unimodal curves, in which both tails dropped to a similar plateau, were the most common subtype ( $64.7 \%$ of unimodals) (Figs. $2 B, 3 A$ ). The other subtypes were unimodal curves with high-frequency $(26.7 \%$ ) (Fig. 3B) or lowfrequency $(8.6 \%)$ (Fig. 3C) tails. These curves had high- or low-frequency tails, respectively, that either reascended or plateaued higher than the other tail of the curve.

To assess the response of the olivocerebellar system as a whole, population curves were generated by averaging the individual cell curves from each experiment $(n=21)$. The population curve shapes reflected the preponderance of unimodal and bimodal type cells, with most population curves being either unimodal or bimodal $(n=19)$ (Fig. 3G,H). The remaining two experiments had multipeaked population curves (Fig. 3I). The average peak frequency (lower frequency peak for bimodal curves), amplitude, and half-area width were calculated excluding the latter two experiments (because defining a primary peak was not possible in these cases). The peak had an average frequency of $9.2 \pm 2.2 \mathrm{~Hz}(n=19)$, amplitude of $18.9 \pm$ $12.0 \%$, and half-area width of $7.5 \pm 2.5$ Hz. Similar values were obtained by averaging across all cells $(n=420)$ instead of by experiment. Of particular note is the similarity between the resonance peak frequency and spontaneous CS oscillation frequency.

Because the simple unimodal and bimodal classes together accounted for $\sim 67 \%$ of the cells and were the dominant influence on the population response curve shape, we characterized frequencyresponse curves of these classes in more detail. To characterize the resonance peak of the unimodal curves, we fit each with a Gaussian (Figs. $2 B, 3 A$, dashed line). Of these unimodal curves, 120 of 172 were reasonably fit according to the $\chi^{2}$ goodness of fit test. Curve parameters were obtained from the fitted equations as described in Materials and Methods. The peak occurred at a frequency of $9.5 \pm 2.3 \mathrm{~Hz}$ and had a total amplitude of $20.9 \pm$ $12.7 \%$, a width of $6.0 \pm 3.8 \mathrm{~Hz}$ (note that this width is different from the half-area width; see Materials and Methods for definition), and a quality factor of $4.4 \pm 4.5$. The asymptote of the curve was $4.2 \pm 4.9 \%$, leading to a maximum total peak amplitude to asymptote ratio of 5.0 . 
The peak frequencies of the unimodal curves were distributed fairly broadly over the tested range $(5.3-16.5 \mathrm{~Hz})$, allowing us to test whether the relative sharpness of the resonance peak varied linearly with frequency (Fig. 4A). It did not. The correlation between quality factor and peak frequency $(r=0.026)$ was not statistically different from 0 ( $p=0.78)$, nor was the slope of the regression line $(p=0.78)$; however, there appear to be subpopulations of cells with resonant frequencies that are $\sim 9$ and $\sim 14 \mathrm{~Hz}$ and display high quality factors, although no linear dependence of quality factor on resonant frequency was observed.

The bimodal curves had two distinct resonance peaks: one between 7 and $11 \mathrm{~Hz}$ and one between 12 and $18 \mathrm{~Hz}$. We refer to the $7-11 \mathrm{~Hz}$ peak as the primary resonance peak, because its frequency closely matched the frequency of spontaneous CS oscillatory activity and the peak frequency of the unimodal curves. The higher frequency peak of the bimodal curves will be referred to as the secondary peak. Gaussians could be fit to the primary and secondary resonance peaks of 53 and 40 bimodal curves, respectively, using the goodness of fit test (Fig. 3D, dashed lines). The average primary and secondary peak frequencies were $8.9 \pm 1.0$ and $15.1 \pm 1.3$ $\mathrm{Hz}$. The average total amplitudes were statistically the same (primary, $15.0 \pm 9.0 \%$; secondary, $15.3 \pm 10.5 \% ; p=0.89)$; however, with regard to other parameters, these peaks differed significantly. The primary peak had a narrower width (primary, $3.6 \pm 2.0$; secondary, $7.6 \pm 2.9 ; p=3.5 \times$ $10^{-9}$ ), a higher quality factor (primary, $5.5 \pm 2.7$; secondary, $3.9 \pm 1.7 ; p=0.0014$ ), and a slightly lower asymptote (primary, $3.0 \pm 2.8 \%$; secondary, $4.8 \pm 4.7 \% ; p=0.038$ ) than the secondary peak. Although the quality factor differed between the two peaks, the values for each individual peak had no statistically significant dependence on peak frequency (Fig. $4 B$ ). In sum, the typical bimodal curve consisted of a sharp low-frequency peak and a broader high-frequency peak. The secondary peak frequency occurred close to, but significantly slower than $(p=$ $\left.2.9 \times 10^{-9}\right)$, double the primary peak frequency $(17.7 \mathrm{~Hz})$.

Because of the similarity in peak frequency, a natural comparison to be made is between the peak of the unimodal curves and the primary peak of the bimodal curves. Statistically significant differences between the two populations were observed based on the parameters obtained from the Gaussian curve fits. Compared with the average unimodal peak, the primary peak of the bimodal curve was shorter in total amplitude $(p=0.001)$, narrower $(p=$ $\left.1.4 \times 10^{-6}\right)$, and had a lower asymptote level $(p=0.047)$.

\section{Modification of IO oscillatory properties alters the transmission of MCtx activity to the cerebellum}

The similarity in frequency of the response curve primary resonance peak to the spontaneous CS oscillation frequency suggests that the oscillatory properties of IO neurons are responsible for the resonance peaks. Other possible explanations exist, however,
B

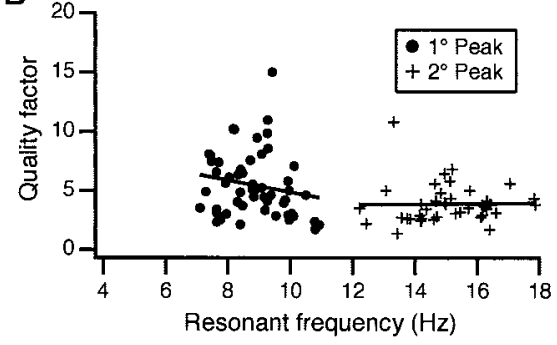


A

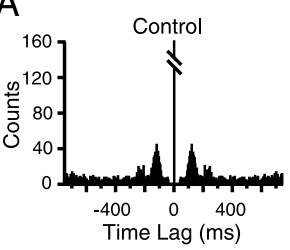

B

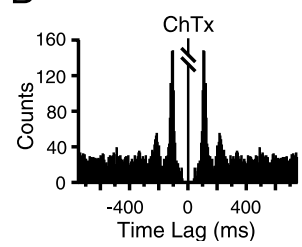

C

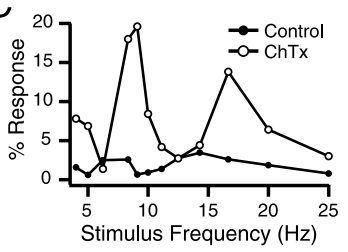

D

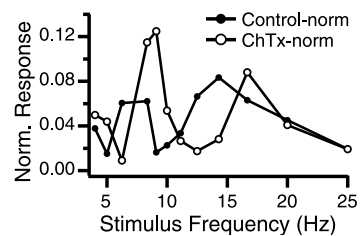

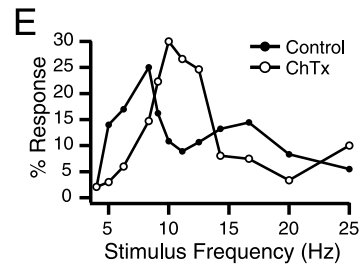

$\mathrm{F}$

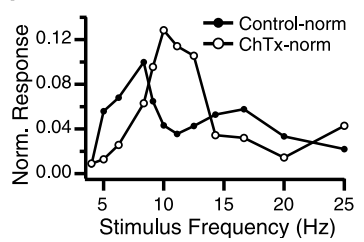

Figure 6. Charybdotoxin selectively enhances $C S$ responses to $\sim 10 \mathrm{~Hz}$ afferent activity from the MCtx. $A, B$, Autocorrelograms of spontaneous $C S$ activity from a Purkinje cell in control $(A)$ and after intra-I0 injection of charybdotoxin $(B)$. Autocorrelograms compiled from $20 \mathrm{~min}$ recording sessions. Bin size $=5 \mathrm{msec}$. Central peaks are truncated. $C-F$, Frequency-response curves in control (filled circles) and charybdotoxin (open circles) conditions. The non-normalized curves for two cells are shown in ( and $E$, with their respective normalized versions in $D$ and $F$.

activity. First, in three of four experiments there was a large increase in overall response rate, which was measured as the total area under the frequency-response curve (control, $71.0 \pm 56.7$; apamin, $354.4 \pm 158.5, n=3$ experiments; $p=5.57 \times 10^{-13}$ ). More importantly, in all four experiments, the shape of the frequency-response curves changed significantly, such that the $\sim 10$ $\mathrm{Hz}$ resonance peak became broader or was lost entirely, as shown by the examples in Figure $5 C-F$.

Because a clear peak in the response curve was often not observed after apamin, we used the peak frequency of the control curve for calculating the apamin curve parameters. Apamin injection caused an increase in the half-area width (control, $7.5 \pm$ $2.9 \mathrm{~Hz}$; apamin, $8.8 \pm 2.3 \mathrm{~Hz} ; p=0.016$ ), consistent with its known effect to weaken IO oscillatory activity. The effect of apamin on the resonance peak amplitude is more difficult to predict because apamin makes IO cells more excitable, which should lead to a general increase in the response probability, but at the same time weakens their oscillatory tendency, which should reduce the amplitude of the resonance peak. Depending on which action is dominant, an increase or decrease in total peak amplitude could occur. In fact, we observed both possibilities. Figure $5 C$ shows a cell in which the peak amplitude decreased despite an overall increase in response rate, whereas for the cell shown in Figure $5 E$, the general increase in response rate dominated, leading to an overall increase in amplitude at the control peak frequency. On average, the weakening of the resonance response appears to have been slightly greater because apamin caused a significant reduction in the mean peak amplitude (control, $25.6 \pm 20.3 \%$; apamin, $17.1 \pm 12.0 \%$; $p=0.021$ ).

Nevertheless, from the examples in Figure 5, $C$ and $E$, it is clear that changes in total peak amplitude do not entirely capture the effect of apamin on the resonance peak. It would be better to measure the change in the relative increase in the $6-12 \mathrm{~Hz}$ range, and to do this, the general increase in excitability caused by apamin needs to be controlled for. Thus, we normalized each frequency-response curve to a total area of 1. Calculation of the peak amplitude from these normalized curves revealed that apamin caused a dramatic reduction in the peak amplitude in every experiment. Overall, the normalized peak amplitudes were reduced to $39.4 \%$ of control levels by apamin (control, $0.142 \pm$ 0.116 ; apamin, $\left.0.056 \pm 0.026 ; p=5.34 \times 10^{-6}\right)$. Figure $5, D$ and
$F$, shows corresponding normalized curves for Figure 5, $C$ and $E$, and demonstrates the reduction in peak amplitude independent of changes in overall response rate.

Consistent with previous findings (Lang et al., 1997), intra-IO injections of charybdotoxin ( $n=43$ cells; 3 experiments) and iberiotoxin ( $n=44$ cells; 2 experiments) increased the excitability and rhythmicity of spontaneous CS activity. Because these drugs produced similar effects, the data were pooled for analysis. Intra-IO injection of these toxins increased the average firing rate (control, $0.76 \pm 0.62$; toxin, $1.71 \pm 1.01 ; p=2.6 \times$ $10^{-11}$ ), oscillation frequency (control, $9.9 \pm 2.7$; toxin, $11.2 \pm 2.1 ; p=0.001)$, average number of autocorrelogram peaks (control, $1.6 \pm 1.0$; toxin, $2.0 \pm 1.3 ; p=$ $0.0058)$, height of the primary autocorrelogram peak (control, $1.9 \pm 0.8 \%$; toxin, $\left.5.1 \pm 4.7 \% ; p=3.8 \times 10^{-8}\right)$, and RI (control, $0.0232 \pm 0.0114$; toxin, $\left.0.0813 \pm 0.1137 ; p=8.8 \times 10^{-8}\right)$. An example of the enhancement in spontaneous CS rhythmicity caused by charybdotoxin is shown by the autocorrelograms of Figure 6, $A$ and $B$.

Injection of charybdotoxin or iberiotoxin altered the frequency-response curves of MCtx-evoked CSs in a manner consistent with their effect on spontaneous CS activity. The resonance peak amplitude increased (control, $23.7 \pm 15.0 \%$; toxin, $29.8 \pm$ $15.6 \% ; p=8.66 \times 10^{-6}$ ), and its half-area width narrowed (control, $8.4 \pm 1.4$; toxin, $\left.6.3 \pm 1.9 ; p=1.8 \times 10^{-14}\right)$. The shift in peak frequency was consistent with that of spontaneous activity but was not significant (control, $9.7 \pm 3.4 \mathrm{~Hz}$; toxin, $10.1 \pm 2.1$; $p=0.33$ ). Like apamin, charybdotoxin increased CS firing rates and therefore may generally have increased IO excitability and responsiveness; however, comparison of curve areas for control and charybdotoxin curves showed that, in fact, this effect was minimal. Overall, the curve areas of the two conditions were not significantly different (control, $251.3 \pm 182.5$; charybdotoxin, $262.2 \pm 164.5 ; p=0.47)$. Nevertheless, to separate a possible effect of general excitability changes from a specific one on the resonance peak, area-normalized curves were generated. Although in some cells the normalization changed the relative increase in peak amplitude (Fig. 6, $C$ vs $D$ ), for most cells the difference was minimal (Fig. 6, $E$ vs $F$ ). Overall, the effect of charybdotoxin on peak amplitude for normalized curves (control, $0.094 \pm 0.027$; charybdotoxin, $0.126 \pm 0.044 ; p=3.78 \times$ $10^{-9}$ ) was similar to what was observed for the non-normalized curves (percentage increase in peak amplitude of 34 versus $26 \%$ ).

In sum, pharmacological manipulations of IO neuronal oscillatory properties produced significant changes in the frequencyresponse curves of CSs evoked by MCtx stimulation and provided evidence that the observed resonance for MCtx-evoked CSs results from the oscillatory properties of IO neurons.

\section{MCtx-evoked CSs display entrainment patterns that reflect the presence of a nonlinear oscillator}

The specific CS response patterns to MCtx stimulation were investigated to provide insight into the nature of the IO oscillator. To begin assessing these patterns, instantaneous firing frequency histograms were made from the CS activity of each cell at each stimulus frequency (the instantaneous firing frequencies of each 
cell were obtained by taking the reciprocals of the interspike intervals during the stimulus trains). The individual cell histograms for each experiment were then summed to generate population histograms. Last, instantaneous firing frequency was converted to entrainment ratio by dividing it by the stimulus frequency (e.g., an instantaneous frequency of $10 \mathrm{~Hz}$ in response to 10 and $20 \mathrm{~Hz}$ stimulus trains would result in entrainment ratios of 1 and 0.5 , respectively).

For most stimulus frequencies, population histograms had multiple peaks with locations that corresponded to integral ratios of the stimulus frequency (i.e., instantaneous frequency/stimulus frequency $=$ $1: 1,1: 2,1: 3$, etc.). Nevertheless, in most cases, one peak was dominant. Thus, the histograms were separated into four categories based on the location of their highest peak, as follows: 1:1, histograms with their largest peak at an entrainment ratio of $1 ; 1: 2$, histograms with their largest peak at an entrainment ratio of 0.5 ; other, histograms with their largest peak at other integral ratios (e.g., 1:3, 1:4, 1:5, etc.); and none, noisy histograms with no prominent peaks, indicating the lack of a dominant entrainment pattern. An example of each of the four categories is shown in Figure $7 A$.

The proportion of population histograms falling into a particular category varied with stimulus frequency. The probability distributions are shown in Figure $7 B$. The distributions indicate that the lowest tested frequencies $(4-5 \mathrm{~Hz})$ often failed to entrain CS activity, with $>60 \%$ of the histograms failing to show a dominant peak in response to $4 \mathrm{~Hz}$ stimulation. Above these frequencies, however, almost all instantaneous frequency histograms had at least one peak indicative of entrainment. It should be noted that the failure of entrainment with 4 and $5 \mathrm{~Hz}$ stimulation is unlikely to have resulted from a failure to activate afferent pathways, because during 4 and $5 \mathrm{~Hz}$ stimulation, the average CS firing rate rose in each case. Overall, the CS firing rate during the $4 \mathrm{sec}$ period preceding 4 or $5 \mathrm{~Hz}$ stimulus trains that did not produce entrainment was $0.8 \pm 0.3 \mathrm{~Hz}$, whereas during those trains it rose to $1.2 \pm 0.4 \mathrm{~Hz}(n=12$ cases from 7 experiments; $p=0.003$ ).

Within the $4-30 \mathrm{~Hz}$ range, at least three distinct entrainment ranges can be defined: low-frequency $(4-6 \mathrm{~Hz})$ and highfrequency $(16-30 \mathrm{~Hz})$ ranges, in which a 1:2 pattern dominated, and separating these two, an intermediate range $(8-16 \mathrm{~Hz})$, in which a 1:1 pattern was dominant. Although one entrainment pattern dominated in each frequency range, it was generally not an absolute dominance because most histograms contained additional smaller peaks at integral ratios of the stimulus frequency. It is also important to note that the borders between these ranges were not absolute. For example, in any particular experiment, the

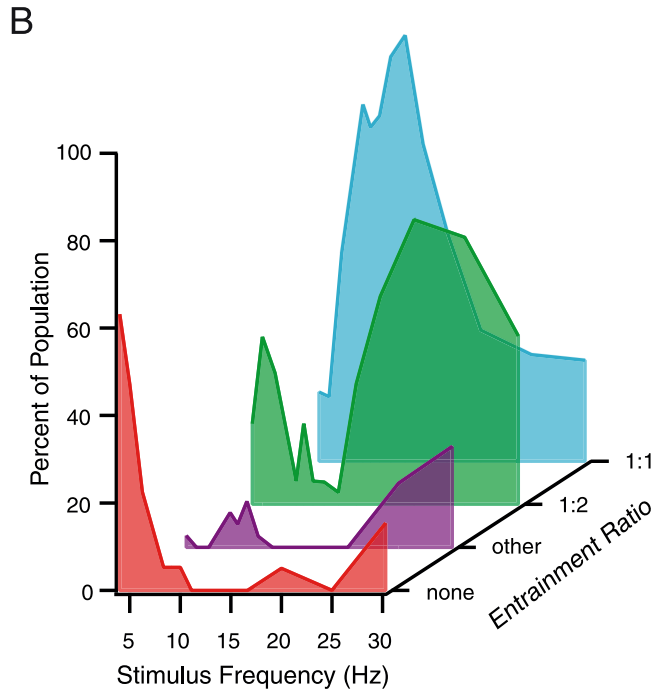

Figure 7. Population instantaneous frequency histogram patterns vary with stimulus frequency. $A$, Instantaneous frequency histograms showing the four main entrainment categories that were observed (1:1, 1:2, other, and none). Instantaneous firing frequency divided by stimulus frequency (entrainment ratio) is plotted on the abscissa of each histogram. Stimulus frequency is 列 grams with a peak at 0.33 (1:3, arrow in second from top histogram), and those with peaks indicating ratios of 1:4 and 1:5. $B$ Distribution of entrainment categories as a function of stimulus frequency. The distributions are color coded to the example Percentages were determined by counting the population histograms in each category for each stimulus frequency. If the two categories were each incremented by a value of one-half. This splitting was necessary for only relatively few cases (16\%; 39 of 239 histograms), and their inclusion did not change the overall distributions significantly.

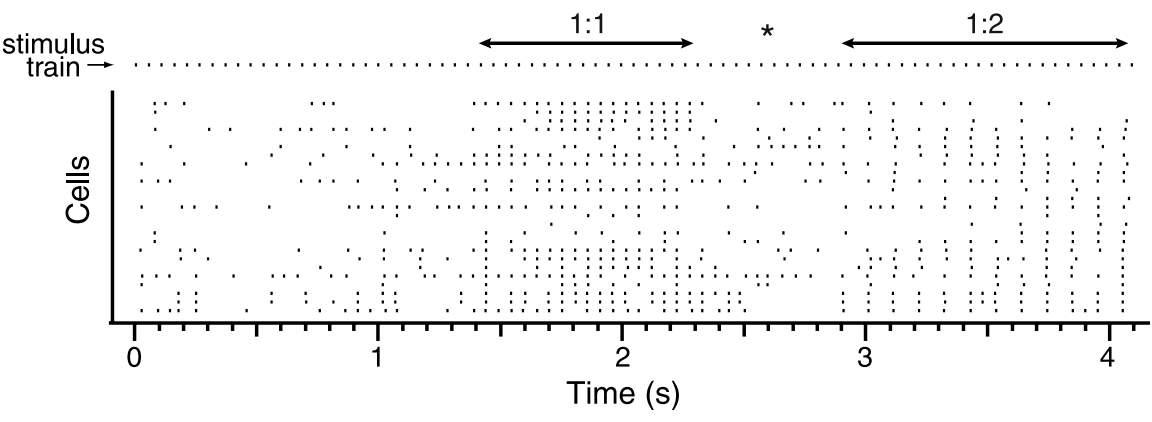

Figure 8. CS responses switch between entrainment patterns during MCtx stimulation. Raster display of CS activity during one $4 \mathrm{sec}, 20 \mathrm{~Hz}$ MCtx stimulus train. Each row represents the CSs from a single Purkinje cell. Stimulus times are shown in top row (stimulus train). During this train, there was one period each of 1:1 and 1:2 entrainment, as indicated on the figure. Asterisk indicates transition period between 1:1 and 1:2 entrainment.

transition from 1:1 to 1:2 dominance could occur at 14, 17, or 20 Hz. Nevertheless, the ranges as specified above reflect the most common transition points.

The entrainment ratio histograms by themselves generally did not guarantee the presence of sustained entrainment patterns, because most had several peaks. For example, a histogram with large peaks at 1 and 0.5 would mean that the cell showed both 1:1 and 1:2 responding; these patterns might be thoroughly intermixed, in which case no sustained entrainment pattern would be present. Examination of the raster records revealed that, in fact, sustained ( $\geq 1 \mathrm{sec}$ ) periods during which one type of response dominated across most of the recorded cells were typical, an example of which is shown in Figure 8. Such prolonged entrainment periods were observed in every experiment to all frequencies (except generally to $4 \mathrm{~Hz}$ ). Moreover, the transitions from one entrainment pattern to another were often marked by a pe- 
A
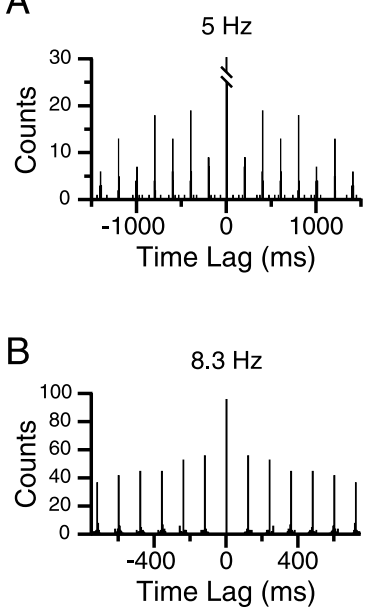
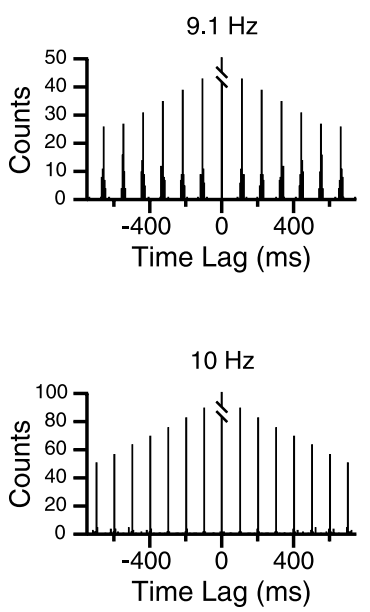
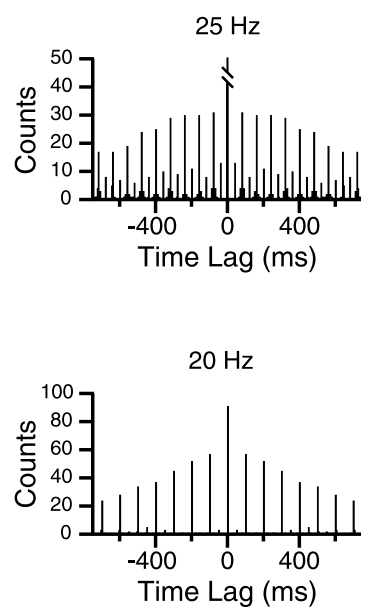

Figure 9. Dominant entrainment pattern varies with stimulus frequency. A, Autocorrelograms of CS activity during MCtx stimulation at various frequencies for one cell. Each autocorrelogram reflects the typical entrainment pattern from one of the three frequency ranges. The 5 and $25 \mathrm{~Hz}$ histograms show alternating peak amplitude patterns characteristic of the low- and highfrequency ranges. The peaks of the $9.1 \mathrm{~Hz}$ histogram gradually decline in amplitude, rather than alternating in size, reflecting 1:1 entrainment. $B$, Autocorrelograms from the CS activity of a second cell during intermediate-frequency $(8.3 \mathrm{and} 10 \mathrm{~Hz})$ and high-frequency $(20 \mathrm{~Hz})$ stimulus trains. Note how the spacing of the peaks in the 10 and $20 \mathrm{~Hz}$ autocorrelograms is identical, because this cell showed only a 1:2 pattern in response to $20 \mathrm{~Hz}$ stimulation. Bin size $=5 \mathrm{msec}$.
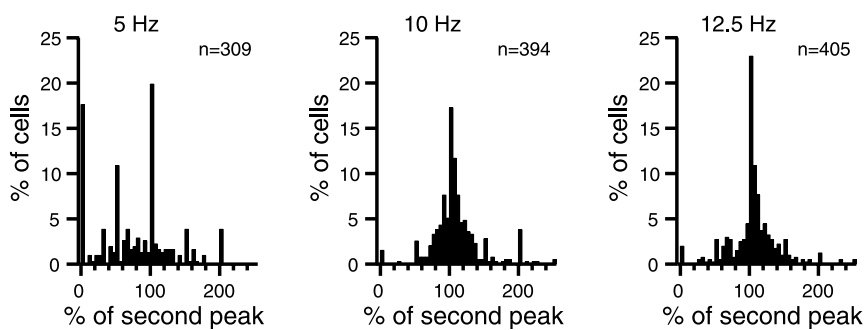

Figure 10. Peak ratio distributions for different stimulus frequencies. The histograms show the distributions of the primary to secondary autocorrelogram peak ratio for CS activity during MCtx stimulation. Each histogram shows the distribution for a different stimulus frequency, indicated above the histogram; $n$ indicates the number of CS autocorrelograms from which the histogram was compiled. Note that for low-frequency $(5 \mathrm{~Hz})$ and high-frequency $(20 \mathrm{~Hz})$ stimulation, a large percentage of cells have a $0 \%$ ratio, whereas for the intermediate frequencies the distribution is centered on the $\sim 100 \%$ bin.

riod of reduced, more irregular firing (Fig. 8, asterisk). Note, also in Figure 8, how the 1:1 and 1:2 patterns occurred at the same times for most cells in the population, which suggests that the entrainment patterns result from IO cells being in distinct oscillatory states rather than from a random skipping of responses to individual stimuli. Such coherent population responses were observed in all experiments.

To quantify further the relative stabilities of entrainment patterns at different stimulus frequencies, single-cell autocorrelograms were generated from the CS activity during trains at each stimulus frequency. Autocorrelograms from two cells are shown in Figure 9, $A$ and $B$. Stimulation at frequencies from 8 to $16 \mathrm{~Hz}$ produced autocorrelograms in which peaks occurred at latencies corresponding to the interstimulus interval (ISI) of the train (Fig. $9 A, 9.1 \mathrm{~Hz}, B, 8.3$ and $10 \mathrm{~Hz}$ ), consistent with the dominant 1:1 peak of most instantaneous frequency histograms at these frequencies (Fig. $7 B$, blue trace). Moreover, neighboring peaks in the autocorrelograms had similar amplitudes, and peaks were typically observed for time lags of at least $1-2 \mathrm{sec}$ (77\% of cells for 9-10 Hz trains).

All autocorrelograms from this intermediate frequency range showed a general trend toward smaller amplitudes with increasingly higher-order peaks, which was the result of several factors.

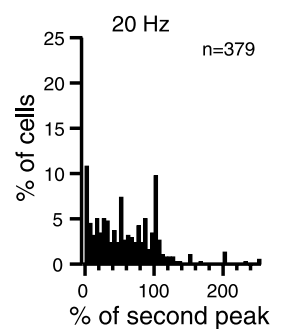

One factor was the finite duration of the spike train; for example, if the cell responded throughout a $4 \mathrm{sec}$ duration, 10 $\mathrm{Hz}$ stimulus train, starting with primary peak (the first peak after the central one at a latency equal to the interstimulus interval), the expected decrease from one peak to the next would be $\sim 2.5 \%$ of the primary peak amplitude (because there are $\sim 40$ stimuli in a train). Steeper declines would reflect increasingly less stable entrainment patterns (e.g., a cell might start responding in the middle of the train, stop responding for periods of time, or show a non-1:1 entrainment pattern during some part of the train).

Thus, the ratio of successive peak amplitudes can be used to measure the stability of the 1:1 entrainment pattern. A ratio of $\sim 100 \%$ (actually slightly $>100 \%$ ) indicates that the entrainment was sustained for a long period, whereas larger ratios indicate a failure to sustain the entrainment. The distribution of the primary to secondary peak amplitude ratio for the entire population is shown in Figure 10, for several stimulus frequencies. For $10 \mathrm{~Hz}$ stimulation, the average primary/secondary peak ratio was near $100 \%$ (110.2 $\pm 38.2 \%$; $n=394)$, indicating that successive MCtx stimuli evoked CS responses with a similar probability, consistent with a predominant 1:1 entrainment at $10 \mathrm{~Hz}$. Similar values were obtained for other stimulus frequencies within the 1:1 entrainment ratio range defined from analysis of the instantaneous frequency histograms. For example, for $12.5 \mathrm{~Hz}$ stimulation, the ratio was $117.2 \pm 69.9(n=405)$ (Fig. 10$)$, and for $14.3 \mathrm{~Hz}$ the average ratio was $102.2 \pm 50.2$ $(n=399)$.

Autocorrelograms from the low $(5-6 \mathrm{~Hz})$ and high $(16-20$ $\mathrm{Hz}$ ) frequency ranges showed a pattern of alternating large and small amplitude peaks superimposed on the gradual trend toward smaller amplitude peaks described for the intermediate frequency range. Moreover, the large amplitude peaks occurred at twice the ISI. Examples of this alternation pattern are shown in Figure $9 A$ ( 5 and $25 \mathrm{~Hz}$ ). Such autocorrelograms reflected the fact that cells had periods of 1:2 and 1:1 responding to high-frequency stimulus trains, consistent with the entrainment ratio histograms from these stimulus frequencies and with raster records, such as shown in Figure 8. In some cases, however, the 1:2 entrainment pattern was completely dominant and resulted in autocorrelograms like those shown in Figure $9 B(20 \mathrm{~Hz})$. Here, the amplitudes of the "small" peaks are close to, or equal, zero (i.e., these peaks are missing), resulting in interpeak intervals of $100 \mathrm{msec}$, twice the ISI.

The primary/secondary peak ratio can be used to quantify the relative dominance of the 1:1 and 1:2 entrainment patterns. As before, the primary peak is the first peak after the central one and occurs at a latency equal to the ISI, and the secondary peak is the second peak after the central one and occurs at a latency of twice the ISI. For perfect 1:2 entrainment, the primary peak equals zero, and therefore the peak ratio will be $0 \%$, whereas as men- 
A. Control
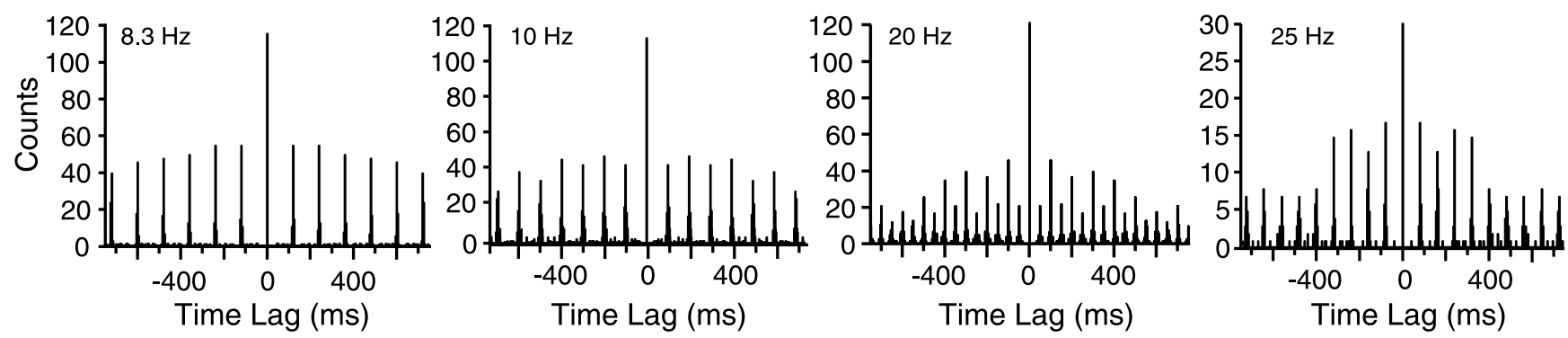

\section{B. Apamin}
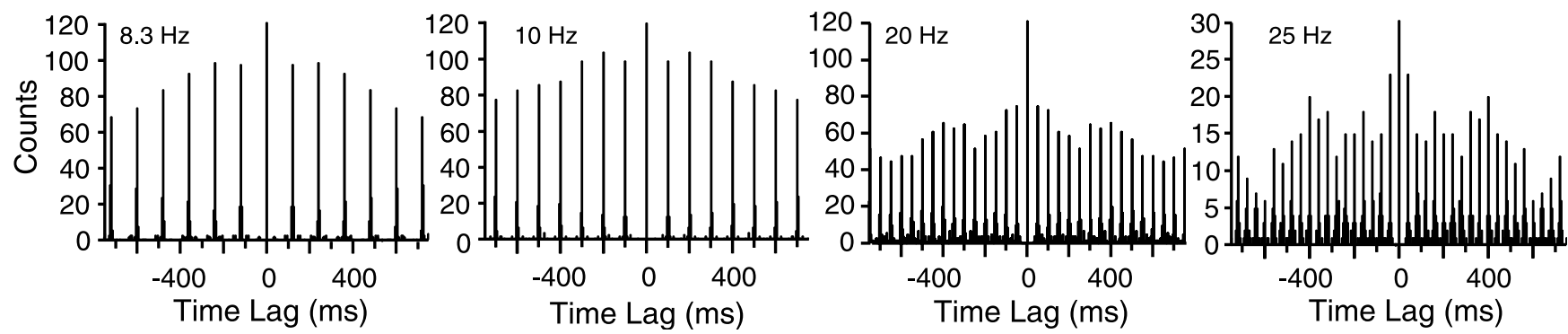

Figure 11. Apamin weakens 1:2 entrainment to high-frequency stimulation. $A, B$, Autocorrelograms of $(S$ activity evoked by $M C t x$ stimulation of different frequencies in control $(A)$ and apamin $(B)$ conditions. Stimulus frequencies are indicated on each autocorrelogram. In control $(A)$, autocorrelograms show typical peak amplitude patterns. After injection of apamin $(B)$, the alternating amplitude patterns to the higher stimulus frequencies are mostly abolished. Bin size $=5 \mathrm{msec}$.

tioned previously, 1:1 entrainment should result in a ratio of $\sim 100 \%$. For cells displaying periods of both 1:1 and 1:2 entrainment (Fig. 8), the ratio will fall between 0 and $100 \%$. The distributions of the peak ratio for low-frequency $(5 \mathrm{~Hz})$ and highfrequency $(20 \mathrm{~Hz})$ stimulation are shown in Figure 10, where they can be compared with those for 10 and $12.5 \mathrm{~Hz}$ stimulation. The peak ratio distributions from the low- and high-frequency ranges were more widespread than those from the intervening frequencies and had significant numbers of cells with ratios of $0 \%$, reflecting almost perfect 1:2 entrainment. Moreover, the average peak ratio values for $5 \mathrm{~Hz}(76.4 \pm 54.1 ; n=309)$ and $20 \mathrm{~Hz}$ $(58.1 \pm 44.0 \% ; n=379)$ were significantly smaller than those from $10,12.5$, or $14.3 \mathrm{~Hz}$ stimulation $(\mathrm{p} \sim 0)$.

Finally, although 1:1 and 1:2 patterns were the most prevalent, the entrainment ratio histograms suggest that cells also could display other, lower ratio (1:3, 1:4, etc.) patterns. These atypical patterns occurred mainly at the higher stimulation frequencies (Fig. 7A, other histogram), although they also could appear at slower frequencies (Fig. $7 B$, purple trace). In addition to being relatively rare, however, these lower ratio entrainments were generally less sustained, and consequently autocorrelogram peak patterns reflecting such entrainments were also less consistent than those reflecting 1:1 and 1:2 entrainment (data not shown).

Modification of IO oscillatory properties alters the strength of the entrainment patterns

The effects of intra-IO injection of apamin, charybdotoxin, and iberiotoxin on the entrainment patterns were investigated to demonstrate that these patterns resulted from the oscillatory properties of IO neurons. Intra-IO injection of apamin reduced the pattern of alternating large and small amplitude peaks typically observed in autocorrelograms of evoked CS activity during high-frequency MCtx stimulation. The effect of apamin injection is shown for one cell in Figure 11. The peak ratios were calculated for 10 and $20 \mathrm{~Hz}$ stimulus trains to quantify the effects of the drug on the entrainment patterns observed in the different frequency ranges. Overall, for $20 \mathrm{~Hz}$ stimulation, apamin significantly increased the first/second peak ratio (control, $54.4 \pm 26.6 \%$; apamin, $74.5 \pm 48.1 \% ; n=47 ; p=0.014$ ) but caused no significant change in the ratio for $10 \mathrm{~Hz}$ stimulation (control, $107.5 \pm$ 23.4\%; apamin, $113.5 \pm 58.3 \% ; n=47 ; p=0.48$ ). Apamin also increased the peak ratio in the low-frequency range. The ratios were calculated for $5 \mathrm{~Hz}$ stimulation, the frequency at which 1:2 entrainment was most dominant within the low-frequency range (Fig. $7 B$ ). Consistent with the $\sim 40 \%$ level in the entire control population, 20 of the 47 cells in the apamin series showed peak ratios $<100 \%$ in control, indicating the presence of 1:2 entrainment. For these cells, apamin basically eliminated the 1:2 pattern, and consequently the average peak ratio increased dramatically from its control value (control, $23.6 \pm 25.3 \%$; apamin, $106.7 \pm$ $\left.61.9 \% ; p=2.6 \times 10^{-5}\right)$.

Intra-IO injection of charybdotoxin or iberiotoxin produced the reverse effect on the entrainment patterns. These drugs enhanced the 1:2 entrainment observed in the high-frequency range. Overall, for $20 \mathrm{~Hz}$ stimulation, the first/second peak ratio decreased from $51.8 \pm 31.6 \%$ in control to $41.8 \pm 38.3 \%$ during charybdotoxin ( $n=75$ cells; $p=0.05$ ), whereas for $10 \mathrm{~Hz}$ stimulation, this ratio was statistically unchanged between the two conditions (control, $107.0 \pm 29.4 \%$; charybdotoxin, $109.7 \pm$ $26.0 \% ; n=82 ; p=0.56)$. An example of the effect of charybdotoxin is shown in Figure 12 for one cell. The autocorrelograms of this cell at 20 and $25 \mathrm{~Hz}$, which had a only modest alternation pattern in control, showed a strong alternation after charybdotoxin injection.

\section{Discussion}

We investigated the frequency dependence of evoked CS responses by using repetitive electrical stimulation of MCtx to drive 


\section{A Control}
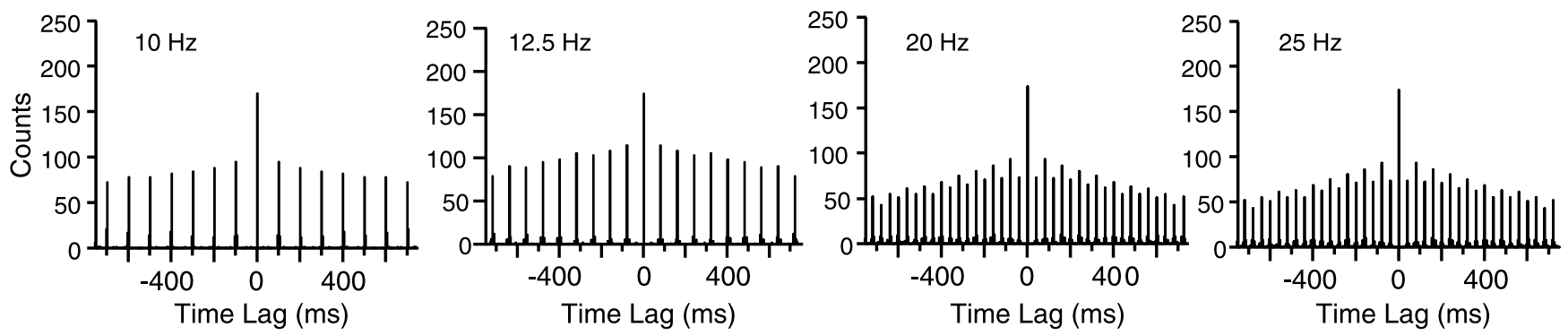

\section{B ChTx}
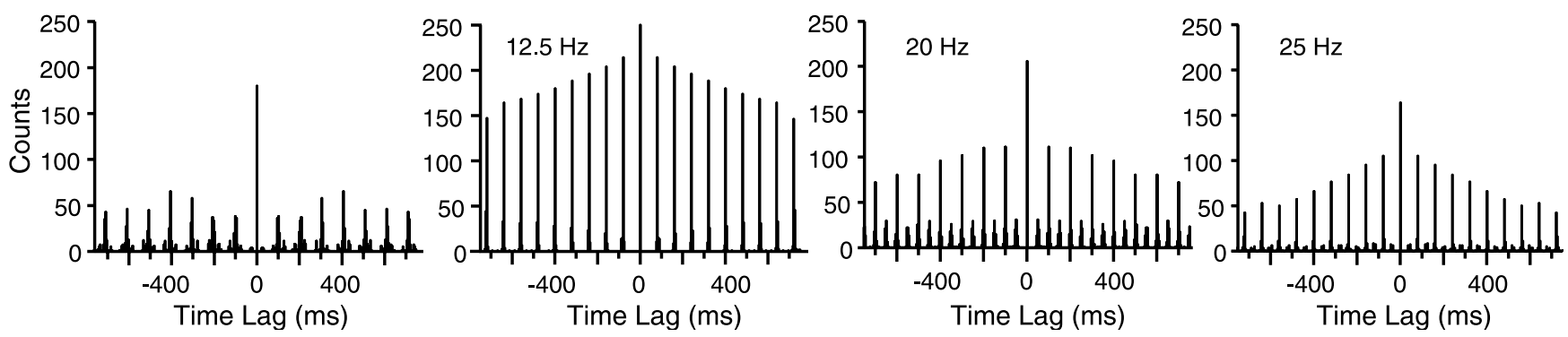

Figure 12. Charybdotoxin enhances 1:2 entrainment to high-frequency stimulation. $A, B$, Autocorrelograms of MCtx-evoked $C S$ activity under control $(A)$ and charybdotoxin $(B)$ conditions. Autocorrelograms show the CS response patterns to stimulus trains of different frequencies (indicated on autocorrelograms) for one cell. This cell showed a relatively modest peak amplitude alternation at 20 and $25 \mathrm{~Hz}$ in control $(A)$. Injection of charybdotoxin greatly enhanced the alternation pattern. Note the near zero amplitude of alternate peaks at $25 \mathrm{~Hz}$. Bin size $=5 \mathrm{msec}$.

the IO in a controlled manner. The observed entrainment patterns and bell-shaped frequency-response curves indicated the presence of an oscillatory modulation of evoked CS responses. Pharmacological manipulations of IO oscillatory ability were shown to alter the entrainment patterns and frequency-response curves, providing evidence that IO neuronal membrane conductances are the source of these phenomena.

\section{CS responses to MCtx activity are shaped by the oscillatory properties of IO neurons}

Several lines of evidence support the conception that the IO functions as a network of coupled oscillators. The olivocerebellar system generates synchronous and rhythmic CS activity (Sasaki et al., 1989; Lang et al., 1999), which remains after IO afferents are blocked (Lang et al., 1996; Lang, 2001, 2003). Moreover, IO neurons display correlated spontaneous subthreshold oscillations in vitro (Llinás and Yarom, 1986).

Previous studies suggest that this oscillatory capability is an important determinant of the response of the olivocerebellar system to afferents (Crill, 1970; Bloedel and Ebner, 1984; Llinás and Sasaki, 1989); however, these studies reported on transient, freerunning responses after single or paired stimuli. Here, we investigated CS responses during sustained rhythmic input. Understanding such responses is important because the IO is likely the recipient of rhythmic activity during normal brain functioning. For example, MCtx displays behavior-related oscillatory activity (Bouyer et al., 1987; Murthy and Fetz, 1992; Sanes and Donoghue, 1993; Nicolelis et al., 1995).

We found that $\sim 90 \%$ of CS frequency-response curves peaked between 6 and $12 \mathrm{~Hz}$. This result is consistent with the facts that oscillators respond strongly to driving frequencies close to their natural one and that IO neurons generally appear to have natural frequencies between 6 and $12 \mathrm{~Hz}$ based on recordings of spontaneous CS activity (Sasaki et al., 1989; Sugihara et al., 1995; Lang et al., 1999) and IO subthreshold oscillations (Llinás and Yarom, 1986; Placantonakis and Welsh, 2001; Devor and Yarom, 2002).

Does this peak reflect a resonance phenomenon caused by the oscillatory properties of IO neurons, or might it reflect greater activation of MCtx-IO pathways with higher stimulus frequencies and a long $(\sim 100 \mathrm{msec})$ refractory period for IO neurons? Several points argue against the latter alternative. First, spontaneous CSs can occur at much shorter intervals $(<40 \mathrm{msec})$. Furthermore, IO neurons can respond to successive stimuli delivered at 33 or $40 \mathrm{msec}$ intervals, as demonstrated by dominant 1:1 entrainment ratios in some cells during 30 and $25 \mathrm{~Hz}$ stimulation (Fig. 7B). Moreover, most cells showing 1:2 entrainment at high stimulus frequencies also had periods of sustained 1:1 responding (Fig. 8). Thus, the refractory period of IO neurons is far shorter than can account for the declines in response probability that start with stimulus frequencies as low as $10 \mathrm{~Hz}$.

Attributing the bimodal and unimodal with high-frequency tail curve shapes to a long, relative refractory period is also problematic. In both cases, frequency ranges above the primary resonance frequency exist in which response probability increases with stimulus frequency. For example, in Figure 3D, the response rate drops from 9 to $14 \mathrm{~Hz}$ but then rises again between 16 and $20 \mathrm{~Hz}$.

Furthermore, drug injection results provide direct evidence that the $8-12 \mathrm{~Hz}$ resonance is related to IO oscillatory properties. Specifically, drugs that altered IO oscillations produced corresponding changes in the resonance peaks of evoked activity. Figure 5 (compare $E, F$ ) demonstrates this point by showing that the $10 \mathrm{~Hz}$ peak is lost despite an overall response increase after apamin.

Last, similar bell-shaped curves were obtained using $\mathrm{pH}$ sensitive dyes to measure olivocerebellar responses to somato- 
sensory stimuli (Hanson et al., 2000), suggesting that the $8-12 \mathrm{~Hz}$ resonance reflects a general response characteristic of olivocerebellar responses rather than the property of a particular afferent.

Given then that MCtx-evoked CS patterns reflect IO oscillatory properties, an important variable should be stimulus phase relative to the IO oscillatory cycle. Because the stimulus train onsets were not linked to the IO cycle, some of them should have occurred out of phase with respect to this cycle. These trains might be expected to evoke few or no CSs. In fact, this type of response was almost never observed (response rates to $10 \mathrm{~Hz}$ trains for all 21 experiments were examined; we found few instances in which individual trains had response rates far below the average rate, and all of these occurred in a single experiment). One explanation for this failure to observe such patterns is that single shocks often reset the IO oscillatory cycle (Llinás and Sasaki, 1989; Manor et al., 2000), and it is likely that the initial stimuli of our trains acted in such a manner. By stepping stimulus intensity lower, one might find a divergence in the response rates to identical frequency stimulus trains.

\section{IO neurons can function as nonlinear, unstable oscillators}

Previous observations suggest that IO neurons act as nonlinear oscillators. For example, they self-generate subthreshold oscillations in vitro (Llinás and Yarom, 1986) and rhythmic CS activity in vivo (Lang, 2001), they can jump between oscillatory and nonoscillatory states (Lang, 2001), their resonant frequency can vary with membrane potential (Llinás and Yarom, 1986), and their spontaneous activity can be modeled by chaotic oscillators (Velarde et al., 2002).

Our results indicate that CS responses to driving stimuli are also characteristic of nonlinear oscillators. For example, CS activity showed 1:1 entrainment to a range of frequencies near its natural frequency, whereas linear oscillators show frequencyspecific beat patterns. Also, the response of a linear oscillator to a driving stimulus is a superposition of a transient response (reflecting the natural frequency of the oscillator) and a steady-state response (reflecting the driving frequency). Thus, initially a linear system will respond at its own frequency, but over time this pattern will be replaced by one reflecting the driving frequency. In contrast, periods of CS responses at the driving frequency (1:1 entrainment) occurred early or late in the stimulus train and could precede or follow periods of $\sim 10 \mathrm{~Hz}$ responses.

Another parameter used to characterize nonlinear oscillators is the stability of their oscillatory states. Initial studies suggested that IO neurons had relatively stable oscillatory states. For example, subthreshold oscillations were reported to be sustained, of constant frequency, and stereotyped for a particular cell (Benardo and Foster, 1986; Llinás and Yarom, 1986; Pettigrew et al., 1988). Moreover, their phase and frequency were unaffected by strong stimuli applied to individual cells (Llinás and Yarom, 1986). Furthermore, after being abolished by extracellular stimuli, oscillations generally reappeared after a short interlude (Benardo and Foster, 1986; Llinás and Yarom, 1986); however, recent results suggest that subthreshold oscillations may not always reflect a stable state because in some IO cells oscillations appeared intermittently and spontaneous shifts in their frequency occurred (Devor and Yarom, 2002).

The present results indicate that, in vivo, most IO neurons have unstable oscillatory states, at least as reflected in CS rhythmicity. In particular, despite CS activity typically becoming entrained during a stimulus train, it did not usually remain so for the duration of the train. Instead, entrainment periods were found to terminate and restart multiple times during a single stimulus train. Autocorrelograms of spontaneous CS activity, which generally show only one to three peaks at $100 \mathrm{msec}$ intervals, also support this conclusion. The percentages of continuous and intermittently oscillating cells in vitro were not given by Devor and Yarom (2002); however, if rhythmic CS activity results from IO subthreshold oscillations, as is often assumed, our results suggest that IO cells should display intermittent subthreshold oscillations in vivo. Parenthetically, supporting the assumption of a relationship between rhythmic CSs and subthreshold IO oscillations, $\sim 5 \mathrm{~Hz}$ subthreshold oscillations can display beat patterns (Lampl and Yarom, 1997) that correspond to the 1:2 entrainment patterns that we observed during low-frequency stimulation.

\section{Do different classes of IO oscillators exist?}

Modeling results suggest that IO neurons can exhibit distinct types of oscillatory behavior (Manor et al., 1997). The various frequency-response curve shapes observed here also support this idea. In particular, bimodal curves differed from unimodal curves in having a second resonance peak and in the parameters of their primary peak. These differences may reflect distinct cell populations or one population with modifiable oscillatory properties.

Bimodal frequency-response curves can result when two oscillators of differing natural frequencies are coupled (Hussey, 1983). This possibility must be considered given the extensive coupling between IO neurons. In this case, however, the frequency-response curve peaks should occur near the two natural frequencies, yet recordings of spontaneous olivocerebellar activity provide no evidence for IO neurons with natural frequencies within most of the secondary bimodal peak frequency range. The peak frequencies of the large majority of unimodal curves also fall below the range for the secondary bimodal peaks, with overlap confined basically to the lower third of the range. Thus, there is no evidence of a population of IO neurons displaying unimodal curves that could underlie the secondary resonance peak of the bimodal curves.

Another possibility is that the unimodal and bimodal curves correspond to different classes of IO neurons. Although possible, the data are more suggestive of a single, continuously varying population (in which the variation is perhaps caused by modulators that control the level of activation of particular ion channels in different IO neurons). Specifically, the amplitudes of the secondary peaks of the bimodal curves formed a continuum in which unimodal curves mark one end. Moreover, drug injections sometimes transformed bimodal curves into unimodal ones (Fig. 6E).

\section{Functional consequences of IO neurons acting as nonlinear oscillators}

The CS frequency-response curves suggest that IO neurons act like bandpass filters; however, for most cells, the curve baseline was above zero, indicating that MCtx neurons can activate the olivocerebellar system over most of their firing range. Nevertheless, for firing rates $>20 \mathrm{~Hz}$, the olivocerebellar response would be relatively weak and insensitive to small fluctuations in rate. In contrast, 8-17 Hz MCtx activity should strongly activate the olivocerebellar system, but perhaps more importantly, the response level should also be very sensitive to changes in MCtx firing rates (because of the steepness of the frequency-response curves in this range). Moreover, the exact range of high sensitivity and efficacy are likely modifiable, because IO neurons can shift their natural frequency within this range (Llinás and Yarom, 1986; Lang et al., 1996; Lang, 2001). Thus, depending on whether 
the frequency content of MCtx activity matches the resonant frequencies of IO neurons, the olivocerebellar system can be attuned to the details of MCtx signals or mostly ignore them.

In sum, the MCtx and IO may dynamically control the strength of their functional connection. Considering that the MCtx interacts with multiple brain areas to plan and execute a movement, the ability to generate various oscillatory rhythms may be a mechanism for allowing the MCtx to select the brain system with which it interacts most strongly at different moments in time.

\section{References}

Allen GI, Azzena GB, Ohno T (1974) Cerebellar Purkinje cell responses to inputs from sensorimotor cortex. Exp Brain Res 20:239-254.

Bal T, McCormick DA (1997) Synchronized oscillations in the inferior olive are controlled by the hyperpolarization-activated cation current $I_{\mathrm{h}}$. J Neurophysiol 77:3145-3156.

Benardo LS, Foster RE (1986) Oscillatory behavior in inferior olive neurons: mechanisms, modulation, cell aggregates. Brain Res Bull 17:773-784.

Bloedel JR, Ebner TJ (1984) Rhythmic discharge of climbing fibre afferents in response to natural peripheral stimuli in the cat. J Physiol (Lond) 352:129-146.

Bouyer JJ, Montaron MF, Vahnée JM, Albert MP, Rougel A (1987) Anatomical localization of cortical beta rhythms in cat. Neuroscience 22:863-869.

Crill WE (1970) Unitary multiple-spiked responses in cat inferior olive nucleus. J Neurophysiol 33:199-209.

Devor A, Yarom Y (2002) Generation and propagation of subthreshold waves in a network of inferior olivary neurons. J Neurophysiol 87:3059-3069.

Hanson CL, Chen G, Ebner TJ (2000) Role of climbing fibers in determining the spatial patterns of activation in the cerebellar cortex to peripheral stimulation: an optical imaging study. Neuroscience 96:317-331.

Hussey M (1983) Fundamentals of mechanical vibrations. New York: Macmillan.

Lampl I, Yarom Y (1993) Subthreshold oscillations of the membrane potential: a functional synchronizing and timing device. J Neurophysiol 70:2181-2186.

Lampl I, Yarom Y (1997) Subthreshold oscillations and resonant behavior: two manifestations of the same mechanism. Neuroscience 78:325-341.

Lang EJ (2001) Organization of olivocerebellar activity in the absence of excitatory glutamatergic input. J Neurosci 21:1663-1675.

Lang EJ (2003) Excitatory afferent modulation of complex spike synchrony. Cerebellum 2:165-170.

Lang EJ, Sugihara I, Llinás R (1996) GABAergic modulation of complex spike activity by the cerebellar nucleoolivary pathway in rat. J Neurophysiol 76:255-275

Lang EJ, Sugihara I, Llinás R (1997) Differential roles of apamin- and charybdotoxin-sensitive $\mathrm{K}^{+}$conductances in the generation of inferior olive rhythmicity in vivo. J Neurosci 17:2825-2838.

Lang EJ, Sugihara I, Welsh JP, Llinás R (1999) Patterns of spontaneous Purkinje cell complex spike activity in the awake rat. J Neurosci 19:2728-2739.

Llinás R, Sasaki K (1989) The functional organization of the olivo-cerebellar system as examined by multiple Purkinje cell recordings. Eur J Neurosci 1:587-602.

Llinás R, Yarom Y (1981a) Electrophysiology of mammalian inferior olivary neurones in vitro. Different types of voltage-dependent ionic conductances. J Physiol (Lond) 315:549-567.

Llinás R, Yarom Y (1981b) Properties and distribution of ionic conductances generating electroresponsiveness of mammalian inferior olivary neurones in vitro. J Physiol (Lond) 315:569-584.

Llinás R, Yarom Y (1986) Oscillatory properties of guinea-pig inferior olivary neurones and their pharmacological modulation: an in vitro study. J Physiol (Lond) 376:163-182.

Manor Y, Rinzel J, Segev I, Yarom Y (1997) Low-amplitude oscillations in the inferior olive: a model based on electrical coupling of neurons with heterogeneous channel densities. J Neurophysiol 77:2736-2752.

Manor Y, Yarom Y, Chorev E, Devor A (2000) To beat or not to beat: a decision taken at the network level. J Physiol (Paris) 94:375-390.

Murthy VN, Fetz EE (1992) Coherent 25- to 35-Hz oscillations in the sensorimotor cortex of awake behaving monkeys. Proc Natl Acad Sci USA 89:5670-5674.

Murthy VN, Fetz EE (1996) Synchronization of neurons during local field potential oscillations in sensorimotor cortex of awake monkeys. J Neurophysiol 76:3968-3982.

Nicolelis MAL, Baccala LA, Lin RCS, Chapin JK (1995) Sensorimotor encoding by synchronous neural ensemble activity at multiple levels of the somatosensory system. Science 268:1353-1358.

Pettigrew AG, Crepel F, Krupa M (1988) Development of ionic conductances in neurons of the inferior olive in the rat: an in vitro study. Proc $\mathrm{R}$ Soc Lond B Biol Sci 234:199-218.

Placantonakis DG, Welsh JP (2001) Two distinct oscillatory states determined by the NMDA receptor in rat inferior olive. J Physiol (Lond) 534:123-140.

Provini L, Redman S, Strata P (1968) Mossy and climbing fibre organization on the anterior lobe of the cerebellum activated by forelimb and hindlimb areas of the sensorimotor cortex. Exp Brain Res 6:216-233.

Ribary U, Ioannides AA, Singh KD, Hasson R, Bolton JPR, Lado F, Mogilner A, Llinás R (1991) Magnetic field tomography of coherent thalamocortical $40-\mathrm{Hz}$ oscillations in humans. Proc Natl Acad Sci USA 88:11037-11041.

Sanes JN, Donoghue JP (1993) Oscillations in local field potentials of the primate motor cortex during voluntary movement. Proc Natl Acad Sci USA 90:4470-4474.

Sasaki K, Bower JM, Llinás R (1989) Multiple Purkinje cell recording in rodent cerebellar cortex. Eur J Neurosci 1:572-586.

Snedecor GW, Cochran WG (1989) Statistical methods, Ed 8. Ames, IA: Iowa State UP.

Steriade M, Hobson JA (1976) Neuronal activity during the sleep-waking cycle. Prog Neurobiol 6:155-376.

Sugihara I, Lang EJ, Llinás R (1995) Serotonin modulation of inferior olivary oscillations and synchronicity: a multiple-electrode study in the rat cerebellum. Eur J Neurosci 7:521-534.

Velarde MG, Nekorin VI, Kazantsev VB, Makarenko V, Llinás R (2002) Modeling inferior olive neuron dynamics. Neural Networks 15:5-10.

Wonnacott TH, Wonnacott RJ (1977) Introductory statistics, Ed 3. New York: Wiley. 